\title{
Uso da Ferramenta Modellus no Ensino de Física: uma aborda- gem a luz da Teoria dos Campos Conceituais
}

\section{The use of Modellus in teaching physics: A light approach to the theory of conceptual fields}

Resumo: Neste artigo relatamos uma experiência com uso da ferramenta computacional Modellus, que é um software livre com fins educacionais desenvolvido para implementação de atividades complementares no ensino de ciências e matemática. Uma sequência didática usando esta ferramenta foi aplicada em duas turmas de 10 Ano do ensino médio em uma escola da rede privada do estado do Rio Grande do Sul. A estratégia de aplicação foi baseada na Teoria dos Campos Conceituais de Vergnaud, que diz que a conceitualização é o centro do desenvolvimento cognitivo. Através de observações feitas durante a aplicação desta proposta, vimos que uma estratégia de ensino baseada no uso de tecnologias como esta, permitem um aprendizado mais eficaz, visto que a interação dos alunos com o objeto de estudo e com as ferramentas educacionais é muito maior quando comparado com as metodologias tradicionais, por isso esta estratégia apresenta um grande potencial como ferramenta para o ensino de Física.

Palavras-chave: Modellus. Modelagem. TIC. Ensino de Física. Campos Conceituais.

Abstract: We report a good experience with the use of Modellus, a freeware computational tool developed for educational purposes generally applied on complementary activities in mathematic and science teaching. A didatical sequence using this tool was performed at two classes of the first period of high school at a private institution in the state of Rio Grande do Sul. Its implementation was based on the Conceptual Fields Theory of Verganaud, whos says that conceptualization is the center of congnitive development. Due to the observations made during the application of this proposal and the results obtained, we saw that a teaching strategy based on the use of technologies such as this allows a more effective learning process, since the contact and interaction of students with the object of study and educational tools is larger when compared with traditional methods, so this strategy has a great potential as an educational resource for teaching Physics.

Keywords: Modellus. Modeling. ICT. Teaching Physics. Conceptual Fields.

ANDRADE, Marcelo Esteves de. Uso da Ferramenta Modellus no Ensino de Física: uma abordagem a luz da Teoria dos Campos Conceituais. Revista Informática na Educação: teoria e prática, Porto Alegre, v. 18, n. 1, p. 27-36, jan./jun. 2015.

\author{
Marcelo Esteves de Andrade \\ Instituto Federal do Espírito Santo
}

\section{Introdução}

N as últimas décadas o ensino de Física na escola secundária tem sido amplamente discutido nos meios acadêmicos e muitas propostas de mudanças têm sido apontadas pelos especialistas da área com o objetivo de que tenhamos uma melhor qualidade no ensino de Física, pois vemos que a metodologia tradicional, uso exclusivo de aulas expositivas e resolução de exercícios, não tem levado os alunos a um desenvolvimento pleno das habilidades que se pretende desenvolver no ensino de Física. Dentre as estratégias apontadas para a melhoria do ensino nesta área destaca-se o uso das novas tecnologias da informação e comunicação (TIC).

Segundo Veit e Teodoro (2002) a utilização destas novas tecnologias ainda está muito defasada em relação ao seu uso científico, necessitando que, para cada área específica do conhecimento, se incorporem as tecnologias no 
processo de ensino-aprendizagem, conforme orientações dos PCNEM (BRASIL, 1999), que por sua vez, também apontam a importância da atualização no ensino em relação às TIC, de forma que o professor seja levado a abordagens e metodologias de ensino mais atuais e eficazes, visando desenvolver as competências e habilidades pelo aluno, em cada disciplina.

Caso não ocorra uma adequação das escolas e dos profissionais da área de educação na produção, desenvolvimento e aplicação de tais tecnologias, o mundo escolar ficará cada vez mais distante do mundo dos alunos (GONÇALVES, 2005).

A utilização das novas tecnologias da informação e comunicação no processo educacional só é justificada se de fato contribuir para a meIhoria e o crescimento na qualidade do ensino e da aprendizagem, por isso a aplicação das tecnologias deve estar em constante avaliação de modo que de fato se verifique a melhoria na construção do conhecimento por parte do aluno, o que de outra forma não justificaria o uso destas novas práticas. Segundo Araújo (2002) dentre as propostas didáticas mais significativas podemos destacar as principais modalidades de uso do computador no ensino de Física: tutoriais (Interactive Journey Through Physics, The Particle Adventure); aquisição de dados (Science Workshop, Real Time Physics, VideoPoint); simulação (Interactive Physics, $x y Z E T$, Graphs and Tracks); modelagem (Stella, Dynamo, PowerSim, Cellular Modelling System, Modellus).

Dentre as modalidades de ferramentas computacionais para o ensino de Física que foram citadas, destacamos aqui a modelagem computacional que tem se mostrado uma ferramenta com um grande potencial para a aprendizagem A modelagem computacional se baseia na construção de um modelo de um fenômeno natural a partir da identificação de va- riáveis e parâmetros envolvidos no fenômeno em questão, e das equações matemáticas que regem tal fenômeno, e a partir desta construção, o modelo pode ser simulado, explorado, testado e também ser expandido para situações mais gerais.

\section{Uso da Modelagem Computa- cional no Ensino de Física e o Pro- grama Modellus}

Dos programas de modelagem disponíveis atualmente, destacamos aqui o Modellus (TEODORO; VIEIRA; CLÉRIGO, 1997). O Modellus é um software de modelagem de distribuição livre para fins educacionais que permite que estudantes e professores simulem de forma dinâmica, diversos fenômenos físicos utilizando modelos matemáticos regidos a partir de funções e equações, apenas escrevendo estas equações de forma direta dentro do programa, ou seja, da mesma forma que o aluno aprendeu a escrever uma equação usando lápis e papel, sem a necessidade de metáforas auxiliares, como no caso de outros programas de modelagem como o Stella, Powersim, e Vensim, por exemplo. O Modellus também permite uma representação variada, de forma que quem usa, pode ver e interagir de forma dinâmica com o modelo criado a partir das equações, das tabelas, das animações e das saídas gráficas que o programa fornece. Numa estratégia didática com o uso de uma ferramenta computacional como esta, o professor passa a ter um outro papel dentro do processo de ensino, que é o papel de conduzir o aluno na construção do conhecimento em física, e não apenas em um transmissor de uma informação que já vem pronta para o aluno, papel este que ao longo dos anos tem sido criticado e se mos- 
trado de pouca utilidade. Nas atividades com o uso da modelagem computacional este papel é modificado, pois o aluno entra em contato direto com o objeto de estudo, e também é levado a atuar de forma direta nas atividades, de modo que o ensino fica centrado nele, cabendo ao professor apenas a condução e a orientação destas atividades. Pensando no potencial destas atividades decidimos criar uma proposta didática mediada pelo o uso desta ferramenta no contexto do ensino de física na escola secundaria, especialmente sobre o tópico da cinemática, que geralmente é abordado no início do primeiro ano do ensino médio.

De acordo com Veit e Teodoro (2002) a introdução da modelagem no processo ensino/ aprendizagem possibilita uma melhor compreensão do seu conteúdo e contribui para o desenvolvimento cognitivo em geral, pois a modelagem facilita a construção de relações e significados, favorecendo a aprendizagem construtivista. Dorneles, Araújo, Dorneles e Veit (2006) destacam que nestas atividades além de poder atuar sobre a variação de parâmetros e valores iniciais, o aluno tem acesso aos elementos básicos do modelo. O aluno pode também, construir seu próprio modelo, desde sua estrutura matemática ou icônica até a análise dos resultados gerados por ele, ou fazer alterações em modelos computacionais previamente construídos. Nestas atividades, que possuem um caráter fortemente interativo, os alunos são levados a fazer generalizações, comparações, testar e modificar variáveis dentro de um dado fenômeno estudado de forma que o aprendizado de Física se torna mais amplo e mais dinâmico. Desta forma, se o aluno é instigado com questões apropriadas, que requeiram interação com o modelo, pode-se favorecer a reflexão deste sobre os efeitos de suas ações sobre os resultados gerados pelo modelo computacional. Dentro deste contexto, o programa de modelagem Modellus se destaca entre outras ferramentas de modelagem devido a sua facilidade de utilização, pois ele não utiliza uma linguagem exclusiva e também pelo fato de ser um programa livre para fins educacionais. Com o Modellus, os alunos podem ser submetidos tanto a atividades exploratórias, que são as atividades onde os alunos têm a possibilidade de alterar os valores de variáveis e parâmetros de um dado modelo pré-estabelecido, como também em atividades expressivas ou de construção, onde dado um certo fenômeno o aluno construirá seu próprio modelo dentro do programa. O Modellus se torna então, uma ferramenta que irá ajudar o professor na construção do conhecimento de Física por parte do estudante.

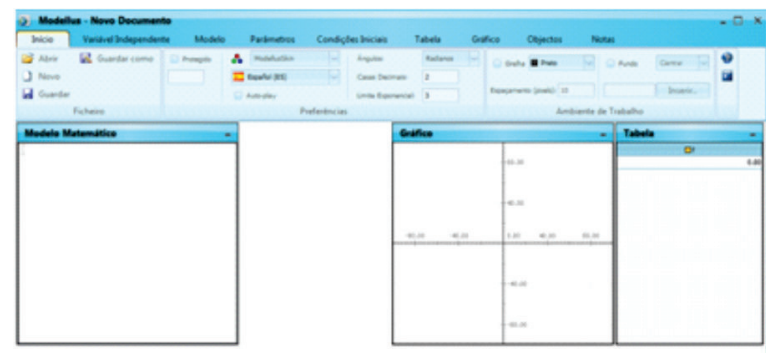

FIGURA 1 - Tela de Apresentação do Software Modellus. FONTE: Acervo Pessoal.

Tais características sugerem que o uso de atividades de modelagem, através do Modellus, pode auxiliar o professor complementando sua prática docente, inclusive em uma escala maior. Araújo (2002) cita também que o fator motivação também é muito importante, pois além do interesse natural despertado pelo 
uso de microcomputadores, os resultados sugerem que a aplicação de atividades de modelagem exerce uma influência positiva na predisposição do indivíduo para aprender Física. Isto ocorre na medida em que a relevância de determinadas relações matemáticas e conceitos é percebida pelo aluno durante o processo de interação com os modelos conceituais, permitindo que o conteúdo visto anteriormente por ele, e que até então estava muito abstrato, passe a ter um referencial mais concreto.

\section{A Teoria dos Campos Conceituais}

Como base para o desenvolvimento de nossa estratégia de ensino usando o programa Modellus, utilizamos a teoria dos Campos Conceituais de Gerard Vergnaud, está teoria é uma teoria cognitivista, que diz que o centro do desenvolvimento cognitivo é a conceitualização (VERGNAUD, 1996b apud MOREIRA, 2002). Esta teoria foi escolhida pois se encaixa muito bem na proposta de ensino abordada, uma vez que escolhemos um caminho onde inicialmente seria trabalhado a parte conceitual subjacente aos conteúdos abordados e em seguida usamos a modelagem computacional como suporte para a análise das situações que trariam sentido aos conceitos.

A teoria de Vergnaud é uma teoria psicológica referente ao processo de conceitualização do real, que possibilita a identificação e o estudo, das continuidades e rupturas entre conhecimentos do ponto de vista de seu conteúdo conceitual (VERGNAUD, 1990 apud MOREIRA, 2002). Vergnaud investiga o desenvolvimento cognitivo a partir da análise do que podemos chamar de sujeito em situação. É o estudo de como o conhecimento se desenvolve no sujeito a partir das situações que ele enfrenta e a partir disto, podemos pesquisar e compreender melhor o desenvolvimento deste sujeito à medida que ele aprende. Vergnaud parte do princípio que o conhecimento está organizado em campos conceituais, e que o domínio destes campos ocorre através de um largo período de tempo (VERGNAUD, 1982 apud MOREIRA, 2002). Um campo conceitual pode ser definido como um conjunto de problemas e situações cujo tratamento requer conceitos, procedimentos e representações de tipos diferentes, mas intimamente relacionados (VERGNAUD, 1983 apud MOREIRA, 2002).

Dentro do estudo das ciências em geral, temos vários exemplos de campos conceituais associados a cada uma das áreas. Na Biologia, por exemplo, temos o campo conceitual da citologia, o da genética, o da botânica. Na Física temos os campos conceituais da Cinemática, da Ondulatória, e da Eletricidade, só para citar alguns.

Vergnaud descreve o seu conceito de campo conceitual a partir de três princípios (VERGNAUD, 1983 apud MOREIRA, 2002):

1. Um conceito não se forma dentro de um só tipo de situação.

2. Uma situação não se analisa com um só conceito.

3. A construção e apropriação de todas as propriedades de um conceito, é um processo que se estende por um longo período de tempo, com analogias e mal entendidos entre situações, concepções, procedimentos e significantes.

A partir disto, para que o indivíduo se desenvolva no que diz respeito à aprendizagem de um campo conceitual, ele deve dominar uma série de conceitos, situações, formas de raciocínio, procedimentos, representações, e 
ações, todas de formas variadas, mas todas elas conectadas. Então, podemos afirmar que na teoria de Vergnaud, o desenvolvimento cognitivo está intimamente relacionado com as situações enfrentadas pelo sujeito e com a conceitualização. Logo, é através de situações e problemas, que os conceitos ganham sentido para o indivíduo. Desta forma Vergnaud define conceito como um conjunto de três elementos, as situações, os invariantes, e as representações simbólicas (VERGNAUD, 1983 apud MOREIRA, 2002).

O conjunto de situações é o que dá sentido ao conceito e ele é chamado de referente do conceito. Os invariantes por sua vez têm a função operacional relativa ao conceito, eles são os objetos, as propriedades e as relações ligadas ao conceito, e são reconhecidos e usados para analisar e dominar as situações do primeiro conjunto. O conjunto dos invariantes é chamado de significado do conceito. 0 conjunto de representações simbólicas envolve a linguagem natural, gráficos, diagramas, e sentenças formais, que podem ser usadas para indicar e representar esses invariantes e, consequentemente, representar as situações e os procedimentos para lidar com elas. Este conjunto é chamado de significante do conceito (VERGNAUD, 1983 apud MOREIRA, 2002).

Já que as situações dão significado aos conceitos, quanto mais situações forem dominadas pelo indivíduo, mais amplo se tornará o sentido deste conceito para ele. Os invariantes por sua vez, tratam das propriedades que definem o objeto e dos procedimentos adotados pelo aluno para resolver as situações. As representações simbólicas permitem que o sujeito se expresse sobre o conceito relacionando o significado com as propriedades dos conceitos. Estes três conjuntos se relacionam então, de forma dinâmica, para que se alcance o de- senvolvimento cognitivo dentro de um campo conceitual (MOREIRA, 2002). Dentro desta relação, surge outro conceito muito importante na teoria de Vergnaud, que é o conceito de esquema. Para Vergnaud, um esquema é a organização do comportamento para uma classe de situações, e é nos esquemas que se deve pesquisar os elementos cognitivos que fazem com que a ação do sujeito seja operatória (VERGNAUD, 1996 apud MOREIRA, 2002). Os esquemas que o sujeito utiliza nas várias situações e os significantes é que dão sentido aos conceitos para esse sujeito (VERGNAUD, 1990 apud MOREIRA, 2002). Como os esquemas se referem ao conjunto de situações, deve-se dar ênfase à interação esquema-situação, pois é através desta interação que o sujeito chega ao entendimento, ou a resolução de uma dada situação, qualquer que seja a sua natureza.

Neste aspecto, a Modelagem Computacional tem um grande potencial para que se alcance este objetivo, pois através dela o professor pode submeter os seus alunos a uma grande variedade de atividades, de forma que muitas situações relativas a um campo conceitual sejam dominadas e assim se chegue ao sentido dos conceitos relativos a este campo conceitual. O preparo e a execução destas atividades podem ser tais, que os alunos, ao usarem seus esquemas para enfrentar determinadas situações, explicitem seu conhecimento de modo que através da mediação do professor estes conhecimentos se tornem verdadeiros conhecimentos científicos.

A teoria dos Campos Conceituais também se enquadra dentro de nossa proposta no que se refere ao papel do professor, que segundo Moreira (2002) é o de conduzir o processo de explicitação do conhecimento implícito do aluno, que por sua vez pode acontecer através de atividades que coloquem o aluno em contato 
com situações de ensino em que o professor faça a mediação entre o aluno e o novo conhecimento que se queira desenvolver.

\section{Aplicação da Proposta de Ensino}

A proposta de foi desenvolvida numa escola da rede privada na cidade de Taquara, Rio Grande do Sul, no ano letivo de 2009 em uma turma do $1^{\circ}$ ano do ensino médio. O tópico de ensino escolhido foi o da cinemática. Em geral este tópico é trabalhado num período de dois meses com três aulas de cinquenta minutos por semana, totalizando em média vinte e quatro aulas ao todo ao longo deste período. A estratégia metodológica foi dividida em duas partes, inicialmente foi trabalhado toda a parte conceitual envolvendo a área da cinemática, que por sua vez foi dividida nos seguintes sub tópicos: Conceitos iniciais, Velocidade, Aceleração, Movimento com velocidade constante, Movimento com aceleração constante. Para cada um destes sub tópicos foram descritos vários objetivos de ensino relacionados aos conceitos envolvidos em cada um deles.

Os conceitos de cada tópico eram então discutidos numa exposição dialogada, sempre buscando uma interação dos alunos ao longo da abordagem. Ao final das discussões de cada tópico os alunos eram submetidos a um teste conceitual, com questões envolvendo os conceitos que acabáramos de analisar. Estes testes serviram com referência para vermos 0 grau de compreensão dos alunos em relação aos conceitos apresentados. Foram destinadas cincos aulas para esta primeira etapa de atividades.

$\mathrm{Na}$ segunda etapa introduzimos então as atividades de modelagem computacional que foram apresentadas a partir de situações- -problema, envolvendo os conceitos e princípios discutidos na parte conceitual. O objetivo principal desta etapa foi o de trabalharmos os referentes dos conceitos que queríamos desenvolver com os alunos, ou seja, as situações que envolviam estes conceitos, e esta análise foi mediada pela modelagem computacional. Para aplicação das atividades de modelagem computacional os alunos foram levados para o laboratório de informática da escola e estes deviam trabalhar sempre em duplas. Destinamos a primeira e a segunda para ensinar os alunos a trabalharem com o programa Modellus. Nestas aulas mostramos aos alunos as principais funções e comandos do programa e como nós iríamos utilizá-los, mostramos a eles como escrever o modelo matemático e como fazer para construir a animação da situação problema proposta.

Quando trabalhamos com a modelagem computacional aplicada ao ensino temos basicamente dois tipos de atividades, as exploratórias e as expressivas. Nas atividades exploratórias, os alunos tem acesso a um modelo matemático e a uma animação que está pronta, e desta forma pode apenas alterar os valores dos parâmetros e de algumas das variáveis do modelo e assim explora-lo de forma dinâmica.

Nas atividades expressivas, ou de construção o aluno deve a partir de uma situação construir o modelo, criar uma animação deste modelo e testa-lo para ver se o resultado condiz com o esperado pela teoria.

Criamos então 10 atividades envolvendo situações problema dos temas abordados, 8 atividades exploratórias e 2 de construção. Cada atividade era realizada em uma aula e foi apresentada a partir de um roteiro, que continha a situação física abordada, instruções para a análise e exploração ou construção do modelo, 
como também questões que deveriam ser respondidas pela dupla com base no que o modelo e a animação mostravam. A figura abaixo mostra um dos modelos de exploração propostos para os alunos.

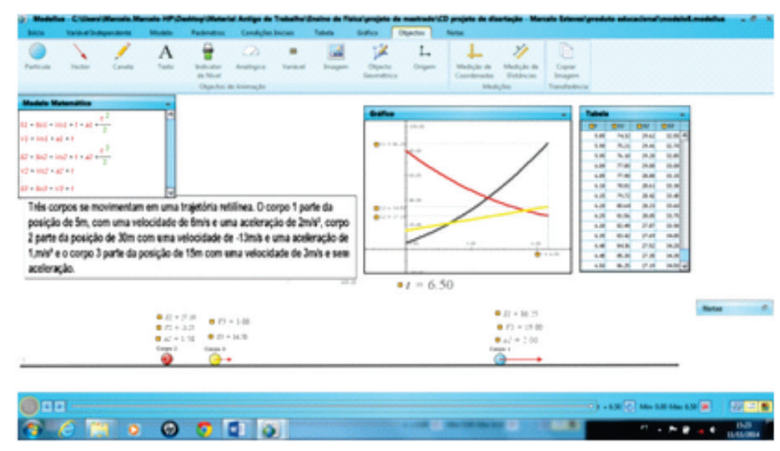

FIGURA 2 - Movimento de 3 Corpos com Aceleração Constante.

FONTE: Acervo Pessoal

Em cada aula as duplas recebiam então o roteiro correspondente ao modelo que seria analisado. Os alunos eram orientados a lerem a situação-problema descrita, analisarem o modelo matemático e só depois rodarem o modelo. Isto fez com que os alunos primeiro tivessem uma noção geral da situação e também do comportamento gerado pelo modelo. Após isto, eles eram levados então para as questões do roteiro.

As questões do roteiro tinham um papel muito importante na aprendizagem, pois era a partir delas que a discussão entre a dupla se iniciava e os conceitos eram então debatidos e posteriormente verificados com a simulação da animação. Ao final de cada aula era feita uma discussão coletiva sobre o modelo analisado e sobre as questões discutidas nos roteiros, desta forma as perguntas que ainda geravam duvidas em algumas duplas eram sanadas.

Nas atividades de construção o roteiro apresentava uma situação física que deveria ser modelada dentro do programa, desta forma os alunos deveriam realizar toda a construção do modelo matemático do fenômeno como também gerar a animação relativa ao modelo. Com estas atividades de modelagem, além de os conceitos ganharem sentido, os alunos tinham a oportunidade de evocar os esquemas que eles desenvolveram ao longo das aulas para resolver as situações dadas, o que Vergnaud (1996 apud MOREIRA, 2002) chama de interação esquema-situação. Quando eles usavam seus esquemas e estes não funcionavam, eles eram levados a buscar outro esquema que desse conta daquela situação específica, isto era feito ou de forma autônoma pela dupla ou também com a ajuda do professor.

Nesta parte do trabalho vale salientar um princípio importante da teoria de Vergnaud (MOREIRA, 2002), que diz que os conceitos em ação e os teoremas em ação (invariantes operatórios) dos estudantes, são conhecimentos implícitos e, que estes têm dificuldades de torná-los explícitos, mas que a explicitação do conhecimento implícito é que pode tornar os teoremas e conceitos em ação em verdadeiros conhecimentos científicos, e nesta etapa o professor tem um papel mediador fundamental.

Nas atividades exploratórias, mas mais ainda nas expressivas, os alunos eram levados a explicitarem o conhecimento que eles adquiriram ao longo das aulas ao interagirem com os modelos e, principalmente, ao responderem as perguntas do roteiro.

Aplicamos mais dois testes ao grupo de alunos, um após a quinta atividade e outro após a decima atividade, cada teste continha dez questões que abordavam os conceitos utilizados nas situações analisadas nos modelos. Os testes mais uma vez serviram de referência para analisarmos o nível de aprendizagem dos alunos. Percebemos que os alunos apresenta- 
ram um aumento de $20 \%$ no nível de acertos, comparados aos testes iniciais.

A partir das observações feitas e da análise de desempenho nos testes, podemos concluir em termos gerais que os objetivos de ensino foram satisfatoriamente alcançados, e desta forma podemos concluir que o uso das ferramentas educacionais empregadas, atrelada à metodologia desenvolvida, fornece mais elementos para que se alcance uma aprendizagem mais efetiva. Pudemos também fazer uma avaliação do desempenho da proposta, principalmente no que se refere ao uso do programa Modellus, a partir da visão dos alunos. Ao final da proposta pedimos que alguns alunos escrevessem um breve comentário sobre as aulas realizadas no laboratório, principalmente nas aulas que usamos o Modellus. Apresentamos abaixo os comentários de 3 alunos, que nos fornecem alguns elementos que também nos indicam as possíveis vantagens no uso desta metodologia.

\section{Aluno 1:}

As atividades foram muito interessantes, pois conseguimos enxergar de forma mais real as trajetórias e os movimentos realizados, e também por que diversificou as atividades, não ficamos só na sala de aula.

Aluno 2:

As atividades realizadas no laboratório de informática foram muito produtivas e atrativas para nós, promovendo um conhecimento mais prático do assunto. Achei o aprendizado eficiente e objetivo, além de ser envolvente. $O$ conteúdo foi bem assimilado e eu entendi muito bem a matéria sobre movimentos.

Aluno 3:

Acho muito importante quando saímos teoria e vamos para a prática. As aulas em que usamos o programa Modellus, com certeza possibilitaram uma maior assimilação do conteúdo, pois praticamos o que vimos.

A partir destes comentários, podemos também perceber a relevância para os alunos da aplicação de uma estratégia de ensino diferenciada, de forma que o envolvimento destes pôde gerar resultados satisfatórios.

As observações que fizemos em sala de aula e a reação dos alunos às atividades computacionais nos mostraram a grande interação que existiu, tanto entre as duplas como também entre as duplas e o professor, o que mostra o grande envolvimento que os alunos tiveram com o conteúdo através das atividades.

Acreditamos que este fator por si só não garante a aprendizagem, mas é um catalisador para a mesma, de modo que facilita o alcance dos objetivos de ensino. A partir dos resultados dos desempenhos dos alunos nas atividades e das observações mencionadas acima, acreditamos que a proposta atingiu o seu objetivo, que foi o de apresentar os conceitos básicos e as situações relacionadas sobre o tópico da cinemática de uma forma atrativa, dinâmica e significativa.

\section{Considerações Finais}

A aplicação de propostas de ensino de Física como esta, baseadas em tecnologias computacionais, aos poucos têm contribuído para a melhoria do ensino nesta disciplina e proporcionado grandes benefícios no que diz respeito ao aprendizado dos alunos, e também na forma como esta disciplina é encarada por eles.

É claro que a implementação de estratégias baseadas em tecnologias não consegue resolver todos os problemas desta área, que por sua vez passa por uma série de fatores, tais 
como a preparação dos professores, a carga horária semanal, o próprio currículo e a escoIha de conteúdos, os recursos e as estruturas que as escolas oferecem.

Mas também fica claro que, o uso destas estratégias traz mudanças substanciais em vários aspectos do processo de ensino, e dão uma cara diferente à disciplina de Física, que geralmente é vista de uma forma negativa pelos alunos, que pelas dificuldades encontradas no domínio desta área acabam desanimando em seu estudo. Esta é uma realidade que nós como professores vemos todos os dias em sala de aula ao aplicarmos a metodologia tradicional, e por vezes estamos tão arraigados a ela, que sentimos uma grande dificuldade em estabelecermos mudanças deste nível.

A proposta de ensino que aplicamos a esta turma de primeiro ano do Ensino Médio para a aprendizagem de cinemática apresentou muitas destas vantagens, o que nos faz concluir que estes recursos têm um potencial muito grande para a aprendizagem e que vale a pena a utilização destes recursos e desta metodologia.
Analisando a nossa proposta de um modo geral, vemos alguns fatores que contribuíram para o sucesso na aprendizagem dos alunos, como o nível de motivação e interesse, a forte interação entre o objeto de estudo e os estudantes e também o papel diferenciado do professor nesta proposta.

O uso contínuo e crescente de tecnologias computacionais hoje no ensino de Física é um processo quase que irreversível, e acreditamos que trabalhos como esse vem mostrar o grande potencial que estes recursos têm, de ajudar na transformação do ensino de Física na educação básica, mudando o papel do professor e do aluno dentro do processo de ensino e também dando um caráter mais dinâmico ao conhecimento.

Esperamos que através deste trabalho, outros professores se interessem e utilizem a modelagem computacional como recurso para as aulas de Física e que num futuro próximo o seu uso esteja mais difundida no ensino de Física do Ensino Médio.

\section{Referências}

ARAUJO, I.S. Um Estudo Sobre o Desempenho de Alunos de Física Usuários da Ferramenta Modellus na Interpretação de Gráficos da Cinemática. 2002. Dissertação (Mestrado em Ensino de Física) - Instituto de Física, Universidade Federal do Rio Grande do Sul, 2002, Porto Alegre, BR-RS.

ARAUJO, I.S.; DORNELES, P.F.T.; VEIT, E.A. Simulação e Modelagem Computacional no Auxílio à Aprendizagem Significativa de Conceitos Básicos de Eletricidade: Parte 1: Circuitos elétricos simples. Revista Brasileira de Ensino de Física, São Paulo, v. 28, n. 4, p. 487-496, set. 2006.

BRASIL. Ministério da Educação. Secretaria de Educação Média e Tecnológica. PCN+- Ensino Médio: orientações educacionais complementares aos Parâmetros Curriculares Nacionais. Brasília, 1999. 
GONÇALVES, L.J. Uso de Animações Visando a Aprendizagem Significativa de Física Térmica no Ensino Médio. 2005. Dissertação (Mestrado Profissional em Ensino de Física) - Instituto de Física, Universidade Federal do Rio Grande do Sul, 2005, Porto Alegre, BR-RS.

MOREIRA, M.A. A Teoria dos Campos Conceituais de Vergnaud, o Ensino de Ciências e a Pesquisa Nesta Área. Investigações em Ensino de Ciências, Porto Alegre, v. 7, n. 1, p. 7-29, 2002.

VEIT, E.A.; TEODORO, V.D. Modelagem no Ensino/Aprendizagem de Física e os Novos Parâmetros Curriculares Para o Ensino Médio. Revista Brasileira de Ensino de Física, São Paulo, v. 24, n. 2, p. 87-96, jun. 2002.

Submetido para avaliação em 21 de novembro de 2014.

Aprovado para publicação em 02 de junho de 2015.

Marcelo Esteves de Andrade - Instituto Federal do Espírito Santo, Cariacica, BR-ES

E-mail: marcelo.andrade@ifes.edu.br 